\title{
AN ABSOLUTELY STABLE PRESSURE-POISSON STABILIZED FINITE ELEMENT METHOD FOR THE STOKES EQUATIONS*
}

\author{
PAVEL BOCHEV ${ }^{\dagger}$ AND MAX GUNZBURGER ${ }^{\ddagger}$
}

\begin{abstract}
The pressure-Poisson stabilized Galerkin method for the Stokes equation requires the choice of a positive parameter. Existing theoretical predictions for the range of parameter values that yield stable discretizations seem to be very pessimistic when compared to the computational evidence. Motivated by this wide gap, we first examine a continuous prototype for this class of schemes. We show that the prototype is absolutely stable; i.e., it is stable for all parameter values, and is optimally accurate. We then define a new, practical variant of the well-known pressurePoisson stabilized scheme. We prove that the new method is absolutely stable just like its continuous prototype and that it achieves optimal convergence rates with respect to the same mesh-independent norms. The new method differs from the standard pressure-Poisson stabilized method in several important aspects. First, its definition does not degrade to a penalty formulation for the lowest order nodal spaces. Second, the method is absolutely stable with respect to the natural norm for the problem, while the standard pressure-Poisson stabilized method is stable with respect to a meshdependent norm.
\end{abstract}

Key words. stabilized finite element methods, mixed methods, Stokes problem

AMS subject classifications. 76D05, 76D07,65F10,65F 30

DOI. $10.1137 /$ S0036142903416547

1. Introduction. The stable and accurate finite element solution of the Stokes problem requires pairs of velocity and pressure spaces that satisfy the inf-sup (or LBB) compatibility condition; see, e.g., $[7,15,16]$. In the past two decades, the formulation of finite element methods that either circumvent or ameliorate this restrictive condition has attracted significant attention. Examples include augmented Lagrangian methods [12], least-squares finite element methods [4], and a group of methods collectively known as consistently stabilized Galerkin methods; see [1, 3, 8, 11, 13, 14, $17,18,19]$. In what follows, we will refer to the members of the latter group as the standard stabilized methods.

In this paper, we develop and analyze a new stabilized formulation that can be related to one of the standard methods originally proposed in [18] and widely known as the pressure-Poisson stabilized Galerkin method. To demonstrate the connection between the new and the standard methods, we introduce the notion of continuous stabilized prototypes. Continuous prototypes are idealized finite element methods that are not necessarily practical. Their role is to provide a template that reveals the proper functional settings and guides the development of practical schemes. In

${ }^{*}$ Received by the editors October 21, 2002; accepted for publication (in revised form) September 10, 2003; published electronically September 18, 2004. This work was performed by an employee of the U.S. Government or under U.S. Government contract. The U.S. Government retains a nonexclusive, royalty-free license to publish or reproduce the published form of this contribution, or allow others to do so, for U.S. Government purposes. Copyright is owned by SIAM to the extent not limited by these rights.

http://www.siam.org/journals/sinum/42-3/41654.html

${ }^{\dagger}$ Computational Mathematics and Algorithms Department, Sandia National Laboratories, Albuquerque, NM 87185-1110 (pbboche@sandia.gov). Sandia is a multiprogram laboratory operated by Sandia Corporation, a Lockheed-Martin Company, for the United States Department of Energy's National Nuclear Security Administration under contract DE-AC-94AL85000.

${ }^{\ddagger}$ School of Computational Science and Information Technology, Florida State University, Tallahassee, FL 32306-4120 (gunzburg@csit.fsu.edu). The research of this author was supported in part by CSRI, Sandia National Laboratories under contract 18407. 
addition, prototypes serve as a gauge to measure the deviation of practical methods from the idealized mathematical setting. All practical methods associated with a particular prototype form a class of methods. Here, we will derive the prototypes that engender the three most commonly used stabilized methods for the Stokes problem. For reasons that will be explained later, we call the three classes the GLS, SGLS, and RGLS method classes.

Consistently stabilized methods contain a positive parameter that must be set to define the method. It is well known that standard stabilized methods can be divided into those that are conditionally stable and those that are absolutely stable, i.e., those that are stable only for a set of restricted values of the parameter and those that are stable for all values of the parameter, respectively. According to previous theoretical analyses, the standard Galerkin least-squares [17] and pressure-Poisson [18] methods fall into the first category while the method of [11] is an example of an absolutely stable method. Stability classifications of stabilized methods are based on sufficient (weak or strong) coercivity conditions for the corresponding forms. Thus, in principle, they represent the worst case scenario and, in practice, there may be a gap between the theoretically predicted stability range of a method and the stability range observed in computational implementations. For the GLS method this gap is very narrow if it exists at all; see [13] or [2]. In other words, for this method, the stability range predicted by existing theory agrees with great accuracy with its practical stability range.

The main focus of this paper will be on the SGLS class which contains the standard pressure-Poisson stabilized method. Our interest in this class is not incidental. In [2], we reported an unusually large discrepancy between the well-known theoretical stability analysis of [8] and the actual, computationally observed stability range of the standard pressure-Poisson Galerkin method. In fact, what was observed computationally indicates that this method is actually absolutely stable. In this paper, we show that there are indeed grounds for such a stability pattern. Most notably, we prove that the continuous SGLS prototype is absolutely stable. Then we define a new discrete member of this class which also turns out to be absolutely stable.

Our new method differs from the standard pressure-Poisson Galerkin formulation in several important aspects. First, its definition does not degrade to a penalty formulation for the lowest-order nodal spaces. Second, we show that our method is absolutely stable with respect to the natural norm on $\mathbf{H}^{1}(\Omega) \times L_{0}^{2}(\Omega)$, while stability of the standard method is with respect to a mesh-dependent norm. Last, while the new method is not fully consistent, it is weakly inconsistent in the sense that finite element approximations converge to all smooth solutions at the best possible rate.

Our analysis suggests that the new, implementable SGLS method is a potentially strong contender in the field of stabilized formulations for the Stokes problem. The absolute stability makes it an attractive alternative to GLS methods that, both theoretically and practically, are known to be only conditionally stable. Compared with the absolutely stable RGLS methods, the new formulation avoids the appearance of local biharmonic terms that in principle should lead to better conditioned matrices. This conjecture is supported by our studies in [2] which suggest that Krylov subspace solvers generally tend to perform better for members of the SGLS family of stabilized methods. Nevertheless, further numerical studies will be needed to reach a definitive conclusion about the practical performance of our new method. These will be reported in a forthcoming paper.

We have organized the paper as follows. In section 2, we summarize notations 
and quote technical results that are used throughout the paper. Section 3 develops the notion of continuous stabilized prototypes starting from a penalized Lagrangian formulation of the Stokes problem. Sections 4 and 5 are the core of this paper. Their focus is on the SGLS class of stabilized methods. In section 4, we consider the continuous prototype of this class and show that it is absolutely stable. Then, in section 5, we proceed to define a new discrete member of the SGLS class and establish its absolute stability and optimal convergence. In section 6 , we conclude the paper with several remarks concerning implementation of the new method.

2. Quotation of results. Let $\Omega$ denote a bounded region in $\mathbb{R}^{n}, n=2,3$, with a Lipschitz continuous boundary $\Gamma=\partial \Omega$. For $p>0, H^{p}(\Omega)$ denotes a Sobolev space of order $p$ with norm and inner product denoted by $\|\cdot\|_{p}$ and $(\cdot, \cdot)_{p}$, respectively. When $p=0$ we use the standard notation $L^{2}(\Omega)$. The symbol $|\cdot|_{k}, 0 \leq k \leq p$, denotes the $k$ th seminorm on $H^{p}(\Omega)$. We recall the subspace $L_{0}^{2}(\Omega)$ of all square integrable functions with vanishing mean and the subspace $H_{0}^{1}(\Omega)$ of all $H^{1}(\Omega)$ functions with vanishing trace. The Poincaré's inequality

$$
C_{P}\|\phi\|_{0} \leq\|\nabla \phi\|_{0} \quad \forall \phi \in H^{p}(\Omega) \cap H_{0}^{1}(\Omega)
$$

implies that the seminorm $|\phi|_{1}=\|\nabla \phi\|_{0}$ is an equivalent norm on $H_{0}^{1}(\Omega)$. Vector analogues of the Sobolev spaces along with vector-valued functions are denoted by upper and lower case bold face font, respectively, e.g., $\mathbf{H}^{1}(\Omega), \mathbf{L}^{2}(\Omega)$, and $\mathbf{u}$. For vectors in Euclidean spaces, we use vector notation, e.g., $\overrightarrow{\mathbf{x}}$ and $\overrightarrow{\mathbf{y}}$. Matrices are denoted by block letters, e.g., $\mathbb{A}$ and $\mathbb{B}$.

$\mathbf{V}^{h}$ and $S^{h}$ will denote a pair of finite element subspaces of $\mathbf{H}_{0}^{1}(\Omega)$ and $L_{0}^{2}(\Omega)$, respectively. We assume that these spaces are defined with respect to the same regular triangulation $\mathcal{T}_{h}$ of the domain $\Omega$ into finite elements $\mathcal{K}$, where $h$ denotes some measure of the grid size. For example, $\mathcal{K}$ can be hexahedrons or tetrahedrons in three dimensions or triangles or quadrilaterals in two dimensions. We will use $C$ to denote a generic constant that is independent of $h$ but whose value may change from place to place. Let $r>0$ and $s>0$ be two integers. It is further assumed that for every $\mathbf{u} \in \mathbf{H}^{r+1}(\Omega)$ and $p \in H^{s+1}(\Omega)$, there exist functions $\mathbf{u}_{I}^{h} \in \mathbf{V}^{h}$ and $p_{I}^{h} \in S^{h}$ such that

$$
\left\|\mathbf{u}-\mathbf{u}_{I}^{h}\right\|_{0}+h\left\|\mathbf{u}-\mathbf{u}_{I}^{h}\right\|_{1} \leq C h^{r+1}\|\mathbf{u}\|_{r+1}
$$

and

$$
\left\|p-p_{I}^{h}\right\|_{0}+h\left\|p-p_{I}^{h}\right\|_{1} \leq C h^{s+1}\|p\|_{s+1},
$$

respectively. We recall the inverse inequalities

$$
\left\|\mathbf{u}^{h}\right\|_{1} \leq C_{I} h^{-1}\left\|\mathbf{u}^{h}\right\|_{0} \quad \text { and } \quad\left\|p^{h}\right\|_{1} \leq C_{I} h^{-1}\left\|p^{h}\right\|_{0}
$$

that hold for finite element spaces on regular triangulations; see [10] or [15].

2.1. Negative norm and inner product. Let $\mathbf{H}^{-1}(\Omega)$ denote the dual of $\mathbf{H}_{0}^{1}(\Omega)$. Using the equivalence of $|\cdot|_{1}$ and $\|\cdot\|_{1}$ on $\mathbf{H}_{0}^{1}(\Omega)$, we equip $\mathbf{H}^{-1}(\Omega)$ with the norm

$$
\|\mathbf{f}\|_{-1}=\sup _{\phi \in \mathbf{H}_{0}^{1}(\Omega)} \frac{(\mathbf{f}, \boldsymbol{\phi})_{0}}{|\phi|_{1}} \quad \forall \mathbf{f} \in \mathbf{H}^{-1}(\Omega) .
$$


The following representation results hold (cf. $[5,6])$.

Lemma 2.1. For all $\mathbf{f} \in \mathbf{H}^{-1}(\Omega)$, we have

$$
\|\mathbf{f}\|_{-1}^{2}=(\mathbf{S f}, \mathbf{f})_{0},
$$

where $\mathbf{S}: \mathbf{H}^{-1}(\Omega) \mapsto \mathbf{H}_{0}^{1}(\Omega)$ is the solution operator for the vector Poisson equation

$$
-\triangle \mathbf{u}=\mathbf{f} \quad \text { in } \Omega \quad \text { and } \quad \mathbf{u}=0 \quad \text { on } \Gamma,
$$

i.e., $\mathbf{u}=\mathbf{S} \mathbf{f}$ if and only if

$$
(\nabla \mathbf{u}, \nabla \mathbf{v})_{0}=(\mathbf{f}, \mathbf{v})_{0} \quad \forall \mathbf{v} \in \mathbf{H}_{0}^{1}(\Omega) .
$$

If $(\cdot, \cdot)_{-1}$ is the inner product associated with $\|\cdot\|_{-1}$, then

$$
(\mathbf{f}, \mathbf{g})_{-1}=(\mathbf{S f}, \mathbf{g})_{0}=(\mathbf{f}, \mathbf{S g})_{0} \quad \forall \mathbf{f}, \mathbf{g} \in \mathbf{H}^{-1}(\Omega) .
$$

Using (2.6), it is not difficult to show that

$$
(-\triangle \mathbf{u}, \mathbf{v})_{-1}=(\mathbf{u}, \mathbf{v})_{0} \quad \forall \mathbf{u} \in \mathbf{H}_{0}^{1}(\Omega), \mathbf{v} \in \mathbf{H}^{-1}(\Omega) .
$$

We also recall the well-known result (cf. [15, p. 20]) that for any connected $\Omega$ there exists a $C_{N}>0$ such that

$$
C_{N}\|p\|_{0} \leq\|\nabla p\|_{-1} \quad \forall p \in L_{0}^{2}(\Omega) .
$$

3. Stabilization of mixed methods for the Stokes problem. We consider the Stokes equations

$$
\begin{aligned}
-\triangle \mathbf{u}+\nabla p=\mathbf{f} & \text { in } \Omega, \\
\nabla \cdot \mathbf{u}=0 & \text { in } \Omega, \\
\mathbf{u}=\mathbf{0} & \text { on } \Gamma .
\end{aligned}
$$

A weak formulation of the Stokes problem is to seek $(\mathbf{u}, p) \in \mathbf{H}_{0}^{1}(\Omega) \times L_{0}^{2}(\Omega)$ such that

$$
\begin{aligned}
A(\mathbf{u}, \mathbf{v})+B(\mathbf{v}, p) & =F(\mathbf{v}) \quad \forall \mathbf{v} \in \mathbf{H}_{0}^{1}(\Omega), \\
B(\mathbf{u}, q) & =0 \quad \forall q \in L_{0}^{2}(\Omega),
\end{aligned}
$$

where $A(\cdot, \cdot), B(\cdot, \cdot)$, and $F(\cdot)$ are defined by

$$
A(\mathbf{u}, \mathbf{v})=\int_{\Omega} \nabla \mathbf{u}: \nabla \mathbf{v} d \Omega, \quad B(\mathbf{v}, p)=-\int_{\Omega} p \nabla \cdot \mathbf{v} d \Omega, \quad \text { and } \quad F(\mathbf{v})=\int_{\Omega} \mathbf{f} \cdot \mathbf{v} d \Omega
$$

respectively. We recall that (3.2)-(3.3) is the optimality system for the saddle-point $(\mathbf{u}, p)$ of the Lagrangian functional

$$
L(\mathbf{v}, q)=\frac{1}{2} A(\mathbf{v}, \mathbf{v})-F(\mathbf{v})+B(\mathbf{v}, q) .
$$

Therefore, the pressure $p$ is the Lagrange multiplier that is introduced into (3.4) to enforce the (weak) incompressibility constraint (3.3). The restriction of (3.2)-(3.3) to a pair of finite element subspaces $\mathbf{V}^{h} \subset \mathbf{H}_{0}^{1}(\Omega)$ and $S^{h} \subset L_{0}^{2}(\Omega)$ yields the Galerkin mixed method: seek $\left(\mathbf{u}^{h}, p^{h}\right) \in \mathbf{V}^{h} \times S^{h}$ such that 


$$
\begin{aligned}
A\left(\mathbf{u}^{h}, \mathbf{v}^{h}\right)+B\left(\mathbf{v}^{h}, p^{h}\right) & =F\left(\mathbf{v}^{h}\right) \quad \forall \mathbf{v}^{h} \in \mathbf{V}^{h}, \\
B\left(\mathbf{u}^{h}, q^{h}\right) & =0 \quad \forall q^{h} \in S^{h} .
\end{aligned}
$$

For continuous pressure approximations and for velocity fields that vanish on the boundary, $B(\cdot, \cdot)$ can be replaced by the equivalent bilinear form

$$
B^{*}(\mathbf{v}, p)=\int_{\Omega} \mathbf{v} \cdot \nabla p d \Omega
$$

It is easy to see that (3.5)-(3.6) is equivalent to the symmetric, indefinite linear algebraic system

$$
\left(\begin{array}{cc}
\mathbb{A} & \mathbb{B}^{T} \\
\mathbb{B} & 0
\end{array}\right)\left(\begin{array}{l}
\overrightarrow{\mathbf{u}} \\
\overrightarrow{\mathbf{p}}
\end{array}\right)=\left(\begin{array}{l}
\overrightarrow{\mathbf{f}} \\
\overrightarrow{\mathbf{0}}
\end{array}\right)
$$

where the elements of $\overrightarrow{\mathbf{u}}$ and $\overrightarrow{\mathbf{p}}$ are the coefficients in the representation in terms of bases of the finite element pair $\left(\mathbf{u}^{h}, p^{h}\right)$; the matrices $\mathbb{A}$ and $\mathbb{B}$ are deduced in the usual manner, using the bases for $\mathbf{V}^{h}$ and $S^{h}$, from the bilinear forms $A(\cdot, \cdot)$ and $B(\cdot, \cdot)$ (or $\left.B^{*}(\cdot, \cdot)\right)$, respectively.

The problems (3.5)-(3.6) and (3.7) are equivalent representations of the optimality system for the saddle-point $\left(\mathbf{u}^{h}, p^{h}\right)$ of (3.4) out of $\mathbf{V}^{h} \times S^{h}$; i.e., they represent a discrete saddle-point problem. As a result, they lead to stable and accurate approximations of $(\mathbf{u}, p)$ if and only if the pair $\left(\mathbf{V}^{h}, S^{h}\right)$ satisfies the following conditions: first, the inf-sup condition (see $[7,15,16]$ ) there exists $C>0$, independent of $h$, such that

$$
\sup _{\mathbf{v}^{h} \in \mathbf{V}^{h}} \frac{B\left(\mathbf{v}^{h}, q^{h}\right)}{\left\|\mathbf{v}^{h}\right\|_{1}} \geq C\left\|q^{h}\right\|_{0} \quad \forall q^{h} \in S^{h},
$$

and second, $A(\cdot, \cdot)$ is coercive on $\mathbf{Z}^{h} \times \mathbf{Z}^{h}$, where $\mathbf{Z}^{h}=\left\{\mathbf{v}^{h} \in \mathbf{V}^{h} \mid B\left(q^{h}, \mathbf{v}^{h}\right)=0 \forall q \in\right.$ $\left.S^{h}\right\}$ is the subspace of discretely solenoidal functions belonging to $\mathbf{V}^{h}$. Examples of unstable pairs include all equal order interpolation spaces defined with respect to the same triangulation of $\Omega$ into finite elements, as well as such combinations as the bilinear-constant pair; see $[15,16]$.

3.1. Continuous stabilized prototypes. In the literature, the term finite element stabilization is commonly applied to describe the application of various regularization techniques either to (3.4) or directly to (3.5)-(3.6) in order to circumvent the inf-sup condition. Stabilization leads to finite element methods that allow for an unrestricted choice of velocity and pressure spaces, including the choice of equal order interpolation. Consistent stabilization is one of the most popular types of regularization because it avoids penalty errors and can, in principle, be extended to achieve an arbitrarily high order of accuracy. Typically, consistently stabilized methods are defined at the discrete level and employ mesh-dependent norms and inner products. In this section, we formulate continuous prototypes for these methods. The prototypes represent idealized variational problems that can be used to derive practical finite element schemes. The origin of the continuous prototypes can be best understood by considering first the regularization of (3.4) by penalty. The relevant penalized Lagrangian functional is

$$
L(\mathbf{v}, q)=\frac{1}{2} A(\mathbf{v}, \mathbf{v})-F(\mathbf{v})+B(\mathbf{v}, q)-\delta\|q\|_{0}^{2} .
$$


The optimality system for (3.8) is to seek $(\mathbf{u}, p) \in \mathbf{H}_{0}^{1}(\Omega) \times L_{0}^{2}(\Omega)$ such that

$$
\begin{gathered}
A(\mathbf{u}, \mathbf{v})+B(\mathbf{v}, p)=F(\mathbf{v}) \quad \forall \mathbf{v} \in \mathbf{H}_{0}^{1}(\Omega), \\
B(\mathbf{u}, q)-\delta M(p, q)=0 \quad \forall q \in L_{0}^{2}(\Omega),
\end{gathered}
$$

where $M(p, q)=(p, q)_{0}$. Thus, the effect emanating from the penalty term in (3.8) is to relax the constraint in (3.3). In terms of algebraic problems, this means that instead of the indefinite problem (3.7), now finite element discretization yields a linear system of the form

$$
\left(\begin{array}{cc}
\mathbb{A} & \mathbb{B} \\
\mathbb{B}^{T} & -\delta \mathbb{M}
\end{array}\right)\left(\begin{array}{l}
\overrightarrow{\mathbf{u}} \\
\overrightarrow{\mathbf{p}}
\end{array}\right)=\left(\begin{array}{l}
\overrightarrow{\mathbf{f}} \\
\overrightarrow{\mathbf{0}}
\end{array}\right)
$$

having a "definite" coefficient matrix. ${ }^{1}$ As a result, one can show that a finite element method based on (3.8) is stable for any conforming choice of $\mathbf{V}^{h}$ and $S^{h}$. The trouble with (3.8) is the penalty error that limits the order of approximation to $O(\sqrt{\delta})$, regardless of the interpolation order of the pair $\left(\mathbf{V}^{h}, S^{h}\right)$.

The idea of consistent stabilization is to modify (3.2) and (3.3) to a problem like (3.9) and (3.10) but without incurring a penalty error. This requires a term that will generate the desired stabilizing contribution but will vanish on all sufficiently smooth exact solutions. To construct such a term, note that thanks to (2.8)

$$
C_{P}\|p\|_{0} \leq\|\nabla p\|_{-1} \leq C\|p\|_{0},
$$

i.e., $\|\nabla p\|_{-1}$ is an equivalent norm on $L_{0}^{2}(\Omega)$. As a result, $\|\nabla p\|_{-1}^{2}$ will have the same stabilization effect as $\|p\|_{0}^{2}$. However, unlike the latter, $\|\nabla p\|_{-1}^{2}$ can be included via the residual of (3.1) and so, when added to (3.2)-(3.3), the term

$$
\delta(-\triangle \mathbf{u}+\nabla p-\mathbf{f},-\alpha \Delta \mathbf{v}+\nabla q)_{-1}
$$

will generate the appropriate stabilizing contribution but without the penalty error. This leads to a family of continuous stabilized prototypes: seek $\left(\mathbf{u}^{h}, p^{h}\right) \in \mathbf{V}^{h} \times S^{h}$ such that

$$
Q_{\alpha}^{\beta}\left(\mathbf{u}^{h}, p^{h} ; \mathbf{v}^{h}, q^{h}\right)=F_{\alpha}^{\beta}\left(\mathbf{v}^{h}, q^{h}\right)
$$

for all $\left(\mathbf{v}^{h}, q^{h}\right) \in \mathbf{V}^{h} \times S^{h}$, where

$$
\begin{aligned}
Q_{\alpha}^{\beta}(\mathbf{u}, p ; \mathbf{v}, q)= & A(\mathbf{u}, \mathbf{v})+B(\mathbf{v}, p)+\beta B(\mathbf{u}, q) \\
& -\delta(-\triangle \mathbf{u}+\nabla p,-\alpha \triangle \mathbf{v}+\beta \nabla q)_{-1}
\end{aligned}
$$

and

$$
F_{\alpha}^{\beta}(\mathbf{v}, q)=F(\mathbf{v})-\delta(\mathbf{f},-\alpha \triangle \mathbf{v}+\beta \nabla q)_{-1}
$$

${ }^{1}$ The matrix in (3.11) is definite in the sense that

$$
\left(\begin{array}{cc}
\mathbb{A} & \mathbb{B} \\
-\mathbb{B}^{T} & +\delta \mathbb{M}
\end{array}\right)
$$

which is obtained from the coefficient matrix in (3.11) by multiplying the lower block of equations by -1 , is real, positive definite. 
are a bilinear form $\left[\mathbf{H}_{0}^{1}(\Omega) \times L^{2}(\Omega)\right]^{2} \mapsto \mathbb{R}$ and a linear functional $\mathbf{H}_{0}^{1}(\Omega) \times L^{2}(\Omega) \mapsto \mathbb{R}$ parametrized by $\alpha, \beta$, and $\delta$. In (3.13) and (3.14), $\alpha$ and $\beta$ take on the values $\{-1,0,1\}$ and $\{-1,1\}$, respectively, and $\delta$ is a positive, real valued parameter. A method is called absolutely stable if the form $Q_{\beta}^{\alpha}$ is weakly or strongly coercive for all values of $\delta$. If this is true only for selected values of $\delta$, the method is called conditionally stable. In what follows, we will work exclusively with continuous pressure approximations, in which case we can write

$$
\begin{aligned}
Q_{\alpha}^{\beta}\left(\mathbf{u}^{h}, p^{h} ; \mathbf{v}^{h}, q^{h}\right) \equiv & A\left(\mathbf{u}^{h}, \mathbf{v}^{h}\right)+B^{*}\left(\mathbf{v}^{h}, p^{h}\right)+\beta B^{*}\left(\mathbf{u}^{h}, q^{h}\right) \\
& -\delta\left(-\triangle \mathbf{u}^{h}+\nabla p^{h},-\alpha \triangle \mathbf{v}^{h}+\beta \nabla q^{h}\right)_{-1} .
\end{aligned}
$$

We call (3.12) prototypes because the $\mathbf{H}^{-1}(\Omega)$ inner product is not computable so that (3.13) or (3.15) and (3.14) cannot be used directly in a finite element method. However, if the $\mathbf{H}^{-1}(\Omega)$ inner product appearing in (3.13) or (3.15) and (3.14) is replaced by a discrete approximation, each prototype will give rise to a practical method. All methods that can be associated with a particular prototype by virtue of such a substitution form the stabilized class generated by this prototype.

Remark 3.1. While the stabilized problem (3.12) is a modification of an equation that represents an optimality system, it is not necessarily itself an optimality system of some modified Lagrangian. Many of the methods defined by (3.12) can only be derived as modifications of (3.5) and (3.6); i.e., they cannot be formulated starting from a modification of (3.4) and then deriving the associated optimality system.

Remark 3.2. If $\mathbf{u}$ is approximated by piecewise linear or bilinear finite element functions, the second order derivative terms in (3.13) vanish and the prototypes (3.12) reduce to a penalized formulation in which the Lagrangian functional (3.4) is penalized by $-\delta\|\nabla q\|_{-1}^{2}$.

Introducing the bilinear forms

$$
D(\mathbf{u}, \mathbf{v})=\delta(-\triangle \mathbf{u},-\triangle \mathbf{v})_{-1}, \quad C(\mathbf{v}, q)=\delta(\triangle \mathbf{v}, \nabla q)_{-1},
$$

and

$$
K(p, q)=\delta(\nabla p, \nabla q)_{-1}
$$

defined on $\mathbf{H}_{0}^{1}(\Omega) \times \mathbf{H}_{0}^{1}(\Omega), \mathbf{H}_{0}^{1}(\Omega) \times L^{2}(\Omega)$, and $L^{2}(\Omega) \times L^{2}(\Omega)$, respectively, we can write (3.15) in the form

$$
\begin{aligned}
Q_{\alpha}^{\beta}\left(\mathbf{u}^{h}, p^{h} ; \mathbf{v}^{h}, q^{h}\right)= & A\left(\mathbf{u}^{h}, \mathbf{v}^{h}\right)+B^{*}\left(\mathbf{v}^{h}, p^{h}\right)+\beta B^{*}\left(\mathbf{u}^{h}, q^{h}\right) \\
& -\alpha D\left(\mathbf{u}^{h}, \mathbf{v}^{h}\right)+\alpha C\left(\mathbf{v}^{h}, p^{h}\right)+\beta C\left(\mathbf{u}^{h}, q^{h}\right)-\beta K\left(p^{h}, q^{h}\right) .
\end{aligned}
$$

It is then easy to see that the discrete system (3.12) is equivalent to a family of linear algebraic systems of the form

$$
\left(\begin{array}{cc}
\mathbb{A}-\alpha \mathbb{D} & \mathbb{B}+\alpha \mathbb{C} \\
\beta(\mathbb{B}+\mathbb{C})^{T} & -\beta \mathbb{K}
\end{array}\right)\left(\begin{array}{l}
\overrightarrow{\mathbf{u}} \\
\overrightarrow{\mathbf{p}}
\end{array}\right)=\left(\begin{array}{l}
\overrightarrow{\mathbf{f}}_{1} \\
\overrightarrow{\mathbf{f}}_{2}
\end{array}\right)
$$

where the matrices $\mathbb{C}, \mathbb{D}$, and $\mathbb{K}$ are respectively deduced in the usual manner from the bilinear forms $C(\cdot, \cdot), D(\cdot, \cdot)$, and $K(\cdot, \cdot)$.

Choosing different $\alpha$ and $\beta$ gives rise to different bilinear forms in (3.13) and to different matrices in (3.16). It is easy to see that the choices $\{\alpha, \beta\}$ and $\{\alpha,-\beta\}$ define variational problems that can be derived from one another by simply changing 
the pressure test function in (3.12) from $q^{h}$ to $-q^{h}$. Likewise, the linear system (3.16) generated by the choice $\{\alpha,-\beta\}$ can be derived from that for the choice $\{\alpha, \beta\}$ by simply scaling the second row of blocks by -1 . Therefore, the linear systems produced by the two choices $\{\alpha, \beta\}$ and $\{\alpha,-\beta\}$ are equivalent in the sense that they have exactly the same solution. ${ }^{2}$ We will call these variational problems, along with their associated bilinear forms and linear algebraic systems, complementary. The choice of $\alpha$ determines the class of complementary forms while the two forms within each class are generated by selecting $\beta$ equal to either 1 or -1 .

For consistency with the established terminology, we call the prototype corresponding to $\alpha=1$ Galerkin least-squares, or GLS. Since taking $\alpha=0$ "simplifies" the weighting function, we call this class of methods simplified Galerkin least-squares, or SGLS. Finally, choosing $\alpha=-1$ "reflects" the sign of the second order term and so we refer to this prototype as reflected Galerkin least-squares, or RGLS.

The standard members of the GLS, SGLS, and RGLS classes of methods are obtained when the $\mathbf{H}^{-1}(\Omega)$ inner product appearing in (3.15) and (3.14) is approximated by a weighted $\mathbf{L}^{2}$ inner product in the following manner:

$$
\begin{aligned}
Q_{\alpha, h}^{\beta}\left(\mathbf{u}^{h}, p^{h} ; \mathbf{v}^{h}, q^{h}\right)= & A\left(\mathbf{u}^{h}, \mathbf{v}^{h}\right)+B^{*}\left(\mathbf{v}^{h}, p^{h}\right)+\beta B^{*}\left(\mathbf{u}^{h}, q^{h}\right) \\
& -\sum_{\mathcal{K} \in \mathcal{T}_{h}} \delta h_{\mathcal{K}}^{2}\left(-\triangle \mathbf{u}^{h}+\nabla p^{h},-\alpha \triangle \mathbf{v}^{h}+\beta \nabla q^{h}\right)_{0, \mathcal{K}}
\end{aligned}
$$

and

$$
F_{\alpha, h}^{\beta}\left(\mathbf{v}^{h}, q^{h}\right)=F\left(\mathbf{v}^{h}\right)-\sum_{\mathcal{K} \in \mathcal{T}_{h}} \delta h_{\mathcal{K}}^{2}\left(\mathbf{f},-\alpha \triangle \mathbf{v}^{h}+\beta \nabla q^{h}\right)_{0, \mathcal{K}},
$$

respectively. When $\alpha=1$ and $\beta=1$, we recover from (3.17) and (3.18) the original GLS method of [17]. For $\alpha=0$ and $\beta=-1$, they give the original pressure-Poisson stabilized mixed method of [18]. The case $\alpha=-1$ and $\beta=1$ gives the method of $[11]$.

The weighted $L^{2}$ norm is not a particularly accurate approximation of the negative norm. Its main defect is that

$$
C_{1}\left(h\left\|\mathbf{u}^{h}\right\|_{0}\right) \leq\left\|\mathbf{u}^{h}\right\|_{-1} \leq C_{2} h^{-1}\left(h\left\|\mathbf{u}^{h}\right\|_{0}\right) .
$$

This equivalence relation, including the factor $h^{-1}$ in the upper bound, is sharp, and means that (3.17) is stable with respect to a mesh-dependent norm that is not uniformly (in $h$ ) equivalent to the norm on $\mathbf{H}^{1}(\Omega) \times L^{2}(\Omega)$. A more sophisticated but also more complicated approximation is to use a discrete equivalent proposed in [6] in the context of least-squares finite element methods. For stabilized methods based on this norm, we refer to [9].

Analyses of the standard GLS and RGLS methods in [17] and [11], respectively, classify the first one as a conditionally stable scheme and the second one as an absolutely stable scheme. This means that for $\alpha=1$, the choice of $\delta$ in (3.17) and (3.18) is restricted to some finite interval $0<\delta_{0} \leq \delta \leq \delta_{\max }$, while for $\alpha=-1$, the form in (3.17) is stable for any positive $\delta$. In both cases, theoretical classifications agree well with the practical stability of the respective finite element methods; see [13] and

\footnotetext{
${ }^{2}$ Although the choices $\{\alpha, \beta\}$ and $\{\alpha,-\beta\}$ yield the same solution, the algebraic properties of the corresponding coefficient matrices can be vastly different. As a result, the performance of iterative solution techniques can also be vastly different; cf. [2].
} 
[2]. However, this is not so for the standard SGLS method. The formal analysis of [8] classified this method as conditionally stable, with a stability range estimate very close to that of the standard GLS method. In practice, after extensive numerical experiments, we found that the standard SGLS behaves much more like the absolutely stabilized RGLS method; see [2]. This unexpected practical stability prompted us to reexamine the SGLS class starting from its continuous prototype. Thus, for the remainder of this paper, our focus will be on SGLS methods.

4. Continuous SGLS. In this section, we show that the continuous SGLS prototype

$$
Q_{0}^{ \pm}(\mathbf{u}, p ; \mathbf{v}, q)=A(\mathbf{u}, \mathbf{v})+B^{*}(\mathbf{v}, p) \pm B^{*}(\mathbf{u}, q)-\delta(-\triangle \mathbf{u}+\nabla p, \pm \nabla q)_{-1}
$$

is absolutely stable.

TheOREM 4.1. Let $\mathbf{V}^{h} \subset \mathbf{H}_{0}^{1}(\Omega)$ and $S^{h} \subset L_{0}^{2}(\Omega) \cap H^{1}(\Omega)$. Then $Q_{0}^{-}(\cdot ; \cdot)$ is coercive for $0<\delta<4$ and $Q_{0}^{ \pm}(\cdot ; \cdot)$ are weakly coercive for any $\delta \geq 4 ;$ i.e., there exists $C>0$, independent of $h$, such that

$$
Q_{0}^{-}\left(\mathbf{u}^{h}, p^{h} ; \mathbf{u}^{h}, p^{h}\right) \geq C\left(\left\|\mathbf{u}^{h}\right\|_{1}^{2}+\left\|p^{h}\right\|_{0}^{2}\right) \quad \forall 0<\delta<4
$$

and

$$
\left.\begin{array}{l}
\sup _{\left(\mathbf{v}^{h}, q^{h}\right) \in \mathbf{V}^{h} \times S^{h}} \frac{Q_{0}^{ \pm}\left(\mathbf{u}^{h}, p^{h} ; \mathbf{v}^{h}, q^{h}\right)}{\left\|\mathbf{u}^{h}\right\|_{1}+\left\|p^{h}\right\|_{0}} \geq C\left(\left\|\mathbf{u}^{h}\right\|_{1}+\left\|p^{h}\right\|_{0}\right) \\
\sup _{\left(\mathbf{v}^{h}, q^{h}\right) \in \mathbf{V}^{h} \times S^{h}} \frac{Q_{0}^{ \pm}\left(\mathbf{v}^{h}, q^{h} ; \mathbf{u}^{h}, p^{h}\right)}{\left\|\mathbf{v}^{h}\right\|_{1}+\left\|q^{h}\right\|_{0}}>0
\end{array}\right\} \quad \forall \delta \geq 4
$$

for any $\left(\mathbf{u}^{h}, p^{h}\right) \in \mathbf{V}^{h} \times S^{h}$.

Proof. Since complementary forms can be obtained from one another by changing the sign of the pressure test functions, it suffices to carry out the proofs for only one of the forms. Here, we choose to work with the minus form $Q_{0}^{-}$. Using (2.7), the stabilizing term in $Q_{0}^{-}$simplifies to

$$
\delta\left(-\triangle \mathbf{u}^{h}+\nabla p^{h}, \nabla q^{h}\right)_{-1}=\delta\left(\left(\mathbf{u}^{h}, \nabla q^{h}\right)_{0}+\left(\nabla p^{h}, \nabla q^{h}\right)_{-1}\right) .
$$

As a result,

$$
Q_{0}^{-}\left(\mathbf{u}^{h}, p^{h} ; \mathbf{v}^{h}, q^{h}\right)=A\left(\mathbf{u}^{h}, \mathbf{v}^{h}\right)+\left(\nabla p^{h}, \mathbf{v}^{h}\right)_{0}+(\delta-1)\left(\nabla q^{h}, \mathbf{u}^{h}\right)_{0}+\delta\left(\nabla p^{h}, \nabla q^{h}\right)_{-1} .
$$

To prove strong the coercivity result, let $\delta$ be a number between 0 and 4 and consider $Q_{0}^{-}\left(\mathbf{u}^{h}, p^{h} ; \mathbf{u}^{h}, p^{h}\right)$. Using Cauchy's inequality and the $\epsilon$ inequality,

$$
\begin{aligned}
Q_{0}^{-}\left(\mathbf{u}^{h}, p^{h} ; \mathbf{u}^{h}, p^{h}\right) & =A\left(\mathbf{u}^{h}, \mathbf{u}^{h}\right)+\delta\left(\nabla p^{h}, \mathbf{u}^{h}\right)_{0}+\delta\left(\nabla p^{h}, \nabla p^{h}\right)_{-1} \\
& \geq\left|\mathbf{u}^{h}\right|_{1}^{2}+\delta\left\|\nabla p^{h}\right\|_{-1}^{2}-\delta\left\|\nabla p^{h}\right\|_{-1}\left|\mathbf{u}^{h}\right|_{1} \\
& \geq\left(1-\frac{\delta}{2 \epsilon}\right)\left|\mathbf{u}^{h}\right|_{1}^{2}+\delta\left(1-\frac{\epsilon}{2}\right)\left\|\nabla p^{h}\right\|_{-1}^{2} .
\end{aligned}
$$

To ensure coercivity, both coefficients above must be positive. Therefore, $\delta$ and $\epsilon$ must satisfy the inequalities

$$
0<\delta<2 \epsilon \quad \text { and } \quad \epsilon<2
$$


This is always possible when $0<\delta<4$. Since $p^{h} \in L_{0}^{2}(\Omega)$ and $\mathbf{u}^{h} \in \mathbf{H}_{0}^{1}(\Omega)$, the final bound

$$
Q_{0}^{-}\left(\mathbf{u}^{h}, p^{h} ; \mathbf{u}^{h}, p^{h}\right) \geq C\left(\delta, C_{P}, C_{N}\right)\left(\left\|\mathbf{u}^{h}\right\|_{1}^{2}+\|p\|_{0}^{2}\right)
$$

follows from (2.8) and (2.1).

To show that $Q_{0}^{-}$is weakly coercive for $\delta \geq 4$, let $\left(\widetilde{\mathbf{v}}^{h}, \widetilde{q}^{h}\right)=\left(\mathbf{u}^{h}, \gamma p^{h}\right)$ for some positive $\gamma$. Then

$$
Q_{0}^{-}\left(\mathbf{u}^{h}, p^{h} ; \widetilde{\mathbf{v}}^{h}, \widetilde{q}^{h}\right)=\left|\mathbf{u}^{h}\right|_{1}^{2}+\gamma \delta\left\|\nabla p^{h}\right\|_{-1}^{2}+(1+\gamma(\delta-1))\left(\nabla p^{h}, \mathbf{u}^{h}\right)_{0} .
$$

Letting $\gamma=1 /(\delta-1)$, the Cauchy and $\epsilon$ inequalities further give

$$
\begin{aligned}
Q_{0}^{-}\left(\mathbf{u}^{h}, p^{h} ; \widetilde{\mathbf{v}}^{h}, \widetilde{q}^{h}\right) & \geq\left|\mathbf{u}^{h}\right|_{1}^{2}+\frac{\delta}{\delta-1}\left\|\nabla p^{h}\right\|_{-1}^{2}-2\left\|\nabla p^{h}\right\|_{-1}\left|\mathbf{u}^{h}\right|_{1} \\
& \geq(1-\epsilon)\left|\mathbf{u}^{h}\right|_{1}^{2}+\left(\frac{\delta}{\delta-1}-\frac{1}{\epsilon}\right)\left\|\nabla p^{h}\right\|_{-1}^{2} .
\end{aligned}
$$

Since $\delta \geq 4$, we can always choose a positive $\epsilon$ such that

$$
\frac{\delta-1}{\delta}<\epsilon<1
$$

This makes both coefficients in the lower bound positive and we can conclude that there exists $C\left(\delta, C_{P}, C_{N}\right)$, independent of $h$, such that

$$
Q_{0}^{-}\left(\mathbf{u}^{h}, p^{h} ; \widetilde{\mathbf{v}}^{h}, \widetilde{q}^{h}\right) \geq C\left(\delta, C_{P}, C_{N}\right)\left(\left\|\mathbf{u}^{h}\right\|_{1}^{2}+\left\|p^{h}\right\|_{0}^{2}\right) .
$$

To complete the proof of the first weak coercivity condition, we note that $\left\|\widetilde{\mathbf{v}}^{h}\right\|_{1}+$ $\left\|\widetilde{q}^{h}\right\|_{0}=\left\|\mathbf{u}^{h}\right\|_{1}+\frac{1}{\delta-1}\left\|p^{h}\right\|_{0}$ so that the last inequality can be recast into

$$
Q_{0}^{-}\left(\mathbf{u}^{h}, p^{h} ; \widetilde{\mathbf{v}}^{h}, \widetilde{q}^{h}\right) \geq C\left(\delta, C_{P}, C_{N}\right)\left(\left\|\mathbf{u}^{h}\right\|_{1}+\left\|p^{h}\right\|_{0}\right)\left(\left\|\widetilde{\mathbf{v}}^{h}\right\|_{1}+\left\|\widetilde{q}^{h}\right\|_{0}\right) .
$$

To prove the second weak coercivity condition, we choose $\mathbf{v}^{h}=-\mathbf{S}\left(\nabla p^{h}\right)$ and $q^{h} \equiv p^{h}$. Using Lemma 2.1

$$
A\left(-\mathbf{S}\left(\nabla p^{h}\right), \mathbf{u}^{h}\right)=-\left(\nabla p^{h}, \mathbf{u}^{h}\right) \quad \text { and } \quad-\triangle\left(-\mathbf{S}\left(\nabla p^{h}\right)\right)=-\nabla p^{h} .
$$

It is now easy to see that

$$
Q_{0}^{-}\left(-\mathbf{S}\left(\nabla p^{h}\right), p^{h} ; \mathbf{u}^{h}, p^{h}\right)=\left(\mathbf{S}\left(\nabla p^{h}\right), \nabla p^{h}\right)_{0}=\left\|\nabla p^{h}\right\|_{-1}^{2}>0,
$$

where the last identity follows again from Lemma 2.1.

It is a straightforward matter to demonstrate that $Q_{0}^{ \pm}$is continuous. Then standard finite element arguments can be used to show that the method is optimally accurate.

Theorem 4.2. Let $(\mathbf{u}, p) \in \mathbf{H}_{0}^{1}(\Omega) \cap \mathbf{H}^{r+1}(\Omega) \times L_{0}^{2}(\Omega) \cap H^{s+1}(\Omega)$ denote a solution of the Stokes problem and let $\left(\mathbf{u}^{h}, p^{h}\right)$ solve $(3.12)$ for $\alpha=0$. Then there exists a constant $C>0$ independent of $h$ such that

$$
\left\|\mathbf{u}-\mathbf{u}^{h}\right\|_{1}+\left\|p-p^{h}\right\|_{0} \leq C\left(h^{r}\|\mathbf{u}\|_{r+1}+h^{s+1}\|p\|_{s+1}\right) .
$$

We note for future reference that the stability and error estimates of the SGLS prototype are given in terms of the natural mesh-independent norm of $\mathbf{H}^{1}(\Omega) \times L^{2}(\Omega)$. 
5. Discrete SGLS. While the continuous SGLS prototype is not a practical method, its analysis hints at a possibility that members of the SGLS family of methods may have far better stability properties than previously thought. In this section we will define a new member of this family that not only is practical but also inherits the absolute stability of its continuous prototype in terms of the same mesh-independent norms. In addition, the new method is also optimally accurate and converges at the same rate as the continuous prototype. To formulate and analyze the new method, we will make use of several discrete operators along with their relevant properties. These are reviewed next.

5.1. Discrete operators. Given a finite element subspace $\mathbf{V}^{h} \subset \mathbf{H}_{0}^{1}(\Omega)$, we define the discrete Laplace operator $-\triangle^{h}$ as the mapping $-\triangle^{h}: \mathbf{H}_{0}^{1}(\Omega) \mapsto \mathbf{V}^{h}$ such that $-\triangle^{h} \mathbf{u}=\mathbf{z}^{h}$ if and only if

$$
\left(\mathbf{z}^{h}, \mathbf{v}^{h}\right)_{0}=\left(\nabla \mathbf{u}, \nabla \mathbf{v}^{h}\right)_{0} \quad \forall \mathbf{v}^{h} \in \mathbf{V}^{h} .
$$

The discrete inverse Laplace operator $\mathbf{S}^{h}$ is the mapping $\mathbf{S}^{h}: \mathbf{H}^{-1}(\Omega) \mapsto \mathbf{V}^{h}$ such that $\mathbf{S}^{h} \mathbf{u}=\mathbf{z}^{h}$ if and only if

$$
\left(\nabla \mathbf{z}^{h}, \nabla \mathbf{v}^{h}\right)_{0}=\left(\mathbf{u}, \mathbf{v}^{h}\right)_{0} \quad \forall \mathbf{v}^{h} \in \mathbf{V}^{h} .
$$

The last operator that we will need is the $L^{2}$ projection operator onto $\mathbf{V}^{h}$. This operator is the mapping $\mathbf{Q}^{h}: \mathbf{L}^{2}(\Omega) \mapsto \mathbf{V}^{h}$ such that $\mathbf{Q}^{h} \mathbf{u}=\mathbf{z}^{h}$ if and only if

$$
\left(\mathbf{z}^{h}, \mathbf{v}^{h}\right)_{0}=\left(\mathbf{u}, \mathbf{v}^{h}\right)_{0} \quad \forall \mathbf{v}^{h} \in \mathbf{V}^{h} .
$$

If the supremum in (2.5) is restricted to the subspace $\mathbf{V}^{h} \subset \mathbf{H}_{0}^{1}(\Omega)$, we obtain the discrete negative seminorm

$$
\|\mathbf{f}\|_{-h}=\sup _{\phi^{h} \in \mathbf{V}^{h}} \frac{\left(\mathbf{f}, \phi^{h}\right)_{0}}{\left|\phi^{h}\right|_{1}} \quad \forall \mathbf{f} \in \mathbf{H}^{-1}(\Omega) .
$$

The next theorem summarizes the properties of the discrete operators and norms that are relevant to our analysis; for part 3, note that

$$
\left\|\left(\mathbf{I}-\mathbf{Q}^{h}\right) \mathbf{u}\right\|_{-k}=\sup _{\boldsymbol{\phi} \in \mathbf{H}_{0}^{k}(\Omega)} \frac{\left(\left(\mathbf{I}-\mathbf{Q}^{h}\right) \mathbf{u}, \boldsymbol{\phi}\right)_{0}}{\|\boldsymbol{\phi}\|_{k}}
$$

where $\mathbf{H}_{0}^{k}(\Omega) \equiv \mathbf{H}^{k}(\Omega) \cap \mathbf{H}_{0}^{1}(\Omega)$.

TheOrem 5.1. 1. For any $\mathbf{f}, \mathbf{g} \in \mathbf{H}^{-1}(\Omega)$, define $(\mathbf{f}, \mathbf{g})_{-h}=\left(\mathbf{S}^{h} \mathbf{f}, \mathbf{g}\right)_{0}=(\mathbf{f}$, $\left.\mathbf{S}^{h} \mathbf{g}\right)_{0}$. Then

$$
\|\mathbf{f}\|_{-h}^{2}=(\mathbf{f}, \mathbf{f})_{-h}
$$

2. For any $\mathbf{u} \in \mathbf{L}^{2}(\Omega)$

$$
\begin{gathered}
\left\|\mathbf{Q}^{h} \mathbf{u}\right\|_{0} \leq C_{I} h^{-1}\|\mathbf{u}\|_{-h}, \\
\|\mathbf{u}\|_{-1}^{2} \leq C\left(h^{2}\|\mathbf{u}\|_{0}^{2}+\|\mathbf{u}\|_{-h}^{2}\right), \\
-\triangle^{h} \cdot \mathbf{S}^{h} \mathbf{u}=\mathbf{Q}^{h} \mathbf{u} .
\end{gathered}
$$


3. For any $\mathbf{u} \in \mathbf{L}^{2}(\Omega)$ and $0<k \leq r+1$

$$
\left\|\left(\mathbf{I}-\mathbf{Q}^{h}\right) \mathbf{u}\right\|_{-k} \leq C h^{k}\|\mathbf{u}\|_{0} .
$$

Proof. For the proof of the characterization (5.5) and the lower equivalence bound (5.7), we refer to [5] or [6]. Here, we will only demonstrate the proofs for the inverse inequality (5.6), the identity (5.8), and the duality estimate (5.9).

Let $\mathbf{u} \in \mathbf{L}^{2}(\Omega)$. Using the definition (5.3) of $\mathbf{Q}^{h}$ in (5.4),

$$
\|\mathbf{u}\|_{-h}=\sup _{\boldsymbol{\phi}^{h} \in \mathbf{V}^{h}} \frac{\left(\mathbf{u}, \phi^{h}\right)_{0}}{\left|\phi^{h}\right|_{1}}=\sup _{\phi^{h} \in \mathbf{V}^{h}} \frac{\left(\mathbf{Q}^{h} \mathbf{u}, \phi^{h}\right)_{0}}{\left|\phi^{h}\right|_{1}} \geq \frac{\left(\mathbf{Q}^{h} \mathbf{u}, \mathbf{Q}^{h} \mathbf{u}\right)_{0}}{\left|\mathbf{Q}^{h} \mathbf{u}\right|_{1}}
$$

Using the first inequality in (2.4) for $\mathbf{Q}^{h} \mathbf{u}$ gives that

$$
\left|\mathbf{Q}^{h} \mathbf{u}\right|_{1} \leq C_{I} h^{-1}\left\|\mathbf{Q}^{h} \mathbf{u}\right\|_{0} .
$$

As a result,

$$
\|\mathbf{u}\|_{-h} \geq \frac{\left\|\mathbf{Q}^{h} \mathbf{u}\right\|_{0}^{2}}{\left|\mathbf{Q}^{h} \mathbf{u}\right|_{1}} \geq \frac{h\left\|\mathbf{Q}^{h} \mathbf{u}\right\|_{0}^{2}}{C_{I}\left\|\mathbf{Q}^{h} \mathbf{u}\right\|_{0}}=h C_{I}^{-1}\left\|\mathbf{Q}^{h} \mathbf{u}\right\|_{0},
$$

which proves (5.6). A straightforward application of (5.1)-(5.3) shows that

$$
\left(-\triangle^{h} \mathbf{S}^{h} \mathbf{u}, \mathbf{v}^{h}\right)=\left(\nabla\left(\mathbf{S}^{h} \mathbf{u}\right), \nabla \mathbf{v}^{h}\right)=\left(\mathbf{u}, \mathbf{v}^{h}\right),
$$

which proves (5.8). To prove (5.9), we use the definition (5.3) of $\mathbf{Q}^{h}$ and Cauchy's inequality to show that

$$
\left(\left(\mathbf{I}-\mathbf{Q}^{h}\right) \mathbf{u}, \boldsymbol{\phi}\right)_{0}=\left(\mathbf{u},\left(\mathbf{I}-\mathbf{Q}^{h}\right) \boldsymbol{\phi}\right)_{0} \leq\|\mathbf{u}\|_{0}\left\|\left(\mathbf{I}-\mathbf{Q}^{h}\right) \boldsymbol{\phi}\right\|_{0}
$$

and then use (2.2) to obtain

$$
\left\|\left(\mathbf{I}-\mathbf{Q}^{h}\right) \phi\right\|_{0} \leq C h^{k}\|\phi\|_{k}
$$

Combining these bounds shows that

$$
\left\|\left(\mathbf{I}-\mathbf{Q}^{h}\right) \mathbf{u}\right\|_{-k} \leq \sup _{\boldsymbol{\phi} \in \mathbf{H}_{0}^{k}(\Omega)} \frac{h^{k} C\|\mathbf{u}\|_{0}\|\boldsymbol{\phi}\|_{k}}{\|\boldsymbol{\phi}\|_{k}}=C h^{k}\|\mathbf{u}\|_{0} .
$$

5.2. An absolutely stable discrete SGLS method. We introduce the bilinear form

$$
\begin{aligned}
Q_{0, h}^{ \pm}\left(\mathbf{u}^{h}, p^{h} ; \mathbf{v}^{h}, q^{h}\right)= & A\left(\mathbf{u}^{h}, \mathbf{v}^{h}\right)+B^{*}\left(\mathbf{v}^{h}, p^{h}\right) \pm B^{*}\left(\mathbf{u}^{h}, q^{h}\right) \\
& -\delta h^{2}\left(-\triangle^{h} \mathbf{u}^{h}+\nabla p^{h}, \pm \nabla q^{h}\right)_{0}
\end{aligned}
$$

and the linear functional

$$
F_{0, h}^{ \pm}\left(\mathbf{v}^{h}, q^{h}\right)=F\left(\mathbf{v}^{h}\right)-\delta h^{2}\left(\mathbf{f}, \pm \nabla q^{h}\right)_{0} .
$$

The new member of the SGLS family of methods is to seek $\left(\mathbf{u}^{h}, p^{h}\right) \in \mathbf{V}^{h} \times S^{h}$ such that

$$
Q_{0, h}^{ \pm}\left(\mathbf{u}^{h}, p^{h} ; \mathbf{v}^{h}, q^{h}\right)=F_{0, h}^{ \pm}\left(\mathbf{v}^{h}, q^{h}\right) \quad \forall\left(\mathbf{v}^{h}, q^{h}\right) \in \mathbf{V}^{h} \times S^{h}
$$


Before we continue with the stability and error analysis of the new method, let us point out that thanks to definition (5.1)

$$
A\left(\mathbf{u}^{h}, \mathbf{v}^{h}\right) \equiv\left(\nabla \mathbf{u}^{h}, \nabla \mathbf{v}^{h}\right)_{0}=\left(-\triangle^{h} \mathbf{u}^{h}, \mathbf{v}^{h}\right)_{0} .
$$

As a result,

$$
\begin{aligned}
Q_{0, h}^{ \pm}\left(\mathbf{u}^{h}, p^{h} ; \mathbf{v}^{h}, q^{h}\right)= & \left(-\triangle^{h} \mathbf{u}^{h}+\nabla p^{h}, \mathbf{v}^{h}\right)_{0} \pm B^{*}\left(\mathbf{u}^{h}, q^{h}\right) \\
& -\delta h^{2}\left(-\triangle^{h} \mathbf{u}^{h}+\nabla p^{h}, \pm \nabla q^{h}\right)_{0} \\
= & \left(-\triangle^{h} \mathbf{u}^{h}+\nabla p^{h}, \mathbf{v}^{h} \mp \delta h^{2} \nabla q^{h}\right)_{0} \pm B^{*}\left(\mathbf{u}^{h}, q^{h}\right)
\end{aligned}
$$

is an equivalent representation of (5.10) and

$$
\left(-\triangle^{h} \mathbf{u}^{h}+\nabla p^{h}, \mathbf{v}^{h} \mp \delta h^{2} \nabla q^{h}\right)_{0} \pm B^{*}\left(\mathbf{u}^{h}, q^{h}\right)=\left(\mathbf{f}, \mathbf{v}^{h} \mp \delta h^{2} \nabla q^{h}\right)_{0}
$$

is an equivalent form of (5.11). Problem (5.13) leads to an interesting interpretation for the new method: it can be viewed as a Petrov-Galerkin-like scheme obtained by modification of the velocity weight function to $\mathbf{v}^{h} \mp \delta h^{2} \nabla q^{h}$.

Because we have replaced $-\triangle \mathbf{u}^{h}$ with $-\triangle^{h} \mathbf{u}^{h}$ in the definition of the method, the term

$$
\left(-\triangle^{h} \mathbf{u}+\nabla p-\mathbf{f}, \mp \delta h^{2} \nabla q^{h}\right)_{0} \neq 0
$$

i.e., the new method, is not, strictly speaking, a consistent formulation. However, as we will see in the next lemma, the inconsistency is very weak. In particular, we will prove that it does not degrade the optimal convergence rate of the method.

Lemma 5.2. Let $(\mathbf{u}, p) \in \mathbf{H}_{0}^{1}(\Omega) \cap \mathbf{H}^{r+1}(\Omega) \times L_{0}^{2}(\Omega) \cap H^{s+1}(\Omega)$ denote a solution of the Stokes problem and let $\left(\mathbf{u}^{h}, p^{h}\right)$ be a solution of (5.11). Then

$$
\begin{aligned}
Q_{0, h}^{ \pm}\left(\mathbf{u}-\mathbf{u}^{h}, p-p^{h} ; \mathbf{v}^{h}, q^{h}\right) & =\delta h^{2}\left(-\triangle \mathbf{u},\left(\mathbf{Q}^{h}-\mathbf{I}\right) \nabla q^{h}\right)_{0} \\
& \leq \delta C h^{r}\|\mathbf{u}\|_{r+1}\left\|q^{h}\right\|_{0}
\end{aligned}
$$

for all $\left(\mathbf{v}^{h}, q^{h}\right) \in \mathbf{V}^{h} \times S^{h}$.

Proof. Consider the minus form. It is easy to see that

$$
\begin{aligned}
Q_{0, h}^{-}\left(\mathbf{u}-\mathbf{u}^{h}, p-p^{h} ; \mathbf{v}^{h}, q^{h}\right) & =\delta h^{2}\left(-\triangle^{h} \mathbf{u}+\nabla p-\mathbf{f}, \nabla q^{h}\right)_{0} \\
& =\delta h^{2}\left(-\left(\triangle^{h}-\triangle\right) \mathbf{u}, \nabla q^{h}\right)_{0} .
\end{aligned}
$$

From the fact that $-\triangle^{h} \mathbf{u} \in \mathbf{V}^{h}$, the definition (5.3) of the $L^{2}$ projection, and the definition (5.1) of $-\triangle^{h}$, it follows that

$$
\left(-\triangle^{h} \mathbf{u}, \nabla q^{h}\right)_{0}=\left(-\triangle^{h} \mathbf{u}, \mathbf{Q}^{h} \nabla q^{h}\right)_{0}=\left(\nabla \mathbf{u}, \nabla \mathbf{Q}^{h} \nabla q^{h}\right)_{0}=\left(-\triangle \mathbf{u}, \mathbf{Q}^{h} \nabla q^{h}\right)_{0}
$$

and so

$$
\left(-\left(\triangle^{h}-\triangle\right) \mathbf{u}, \nabla q^{h}\right)_{0}=\left(-\triangle \mathbf{u},\left(\mathbf{Q}^{h}-\mathbf{I}\right) \nabla q^{h}\right)_{0}
$$

so that the equality in (5.14) is proved. Next, with the help of (5.9) and the inverse inequality (2.4), we have

$$
\begin{aligned}
\left(-\triangle \mathbf{u},\left(\mathbf{Q}^{h}-\mathbf{I}\right) \nabla q^{h}\right)_{0} & \leq\|\triangle \mathbf{u}\|_{r-1}\left\|\left(\mathbf{Q}^{h}-\mathbf{I}\right) \nabla q^{h}\right\|_{1-r} \\
& \leq C h^{r-1}\|\mathbf{u}\|_{r+1}\left\|\nabla q^{h}\right\|_{0} \\
& \leq C h^{r-2}\|\mathbf{u}\|_{r+1}\left\|q^{h}\right\|_{0}
\end{aligned}
$$

from which the inequality in (5.14) follows. 
5.3. Stability and convergence. The main results of this section are to show that the method (5.11) is absolutely stable and that finite element solutions of (5.11) converge at optimal rates. We begin by establishing the absolute stability of the method, i.e., that the bilinear form (5.10) is weakly coercive for all values of the parameter $\delta$. The proof relies upon a technical result presented in the next lemma.

Lemma 5.3. For any $q^{h} \in S^{h}$

$$
\left\|\nabla q^{h}\right\|_{-1}^{2} \leq C\left(h^{2}\left\|\left(\mathbf{I}-\mathbf{Q}^{h}\right) \nabla q^{h}\right\|_{0}^{2}+\left\|\nabla q^{h}\right\|_{-h}^{2}\right) .
$$

Proof. Since we restrict attention to continuous pressure approximations, $\nabla q^{h} \in$ $L^{2}(\Omega)$. Therefore, (5.7) from Theorem 5.1 implies that

$$
\left\|\nabla q^{h}\right\|_{-1}^{2} \leq C\left(h^{2}\left\|\nabla q^{h}\right\|_{0}^{2}+\left\|\nabla q^{h}\right\|_{-h}^{2}\right) .
$$

Adding and subtracting $\mathbf{Q}^{h} \nabla q^{h}$ to the first term and using the triangle inequality give the upper bound

$$
\left\|\nabla q^{h}\right\|_{-1}^{2} \leq C\left(h^{2}\left\|\left(\mathbf{I}-\mathbf{Q}^{h}\right) \nabla q^{h}\right\|_{0}^{2}+h^{2}\left\|\mathbf{Q}^{h} \nabla q^{h}\right\|_{0}^{2}+\left\|\nabla q^{h}\right\|_{-h}^{2}\right) .
$$

The lemma follows by using the inverse inequality (5.6) to bound $h^{2}\left\|\mathbf{Q}^{h} \nabla q^{h}\right\|_{0}^{2}$ by $C_{I}\left\|\nabla q^{h}\right\|_{-h}^{2}$.

TheOREM 5.4. Assume that $\mathbf{V}^{h} \subset \mathbf{H}_{0}^{1}(\Omega)$ and $S^{h} \subset L_{0}^{2}(\Omega) \cap H^{1}(\Omega)$. Then, for any $\delta>0$, there exists a positive constant $C(\delta)$, independent of $h$, such that

$$
\begin{aligned}
\sup _{\left(\mathbf{v}^{h}, q^{h}\right) \in \mathbf{V}^{h} \times S^{h}} \frac{Q_{0, h}^{ \pm}\left(\mathbf{u}^{h}, p^{h} ; \mathbf{v}^{h}, q^{h}\right)}{\left\|\mathbf{v}^{h}\right\|_{1}+\left\|q^{h}\right\|_{0}} & \geq C(\delta)\left(\left\|\mathbf{u}^{h}\right\|_{1}+\left\|p^{h}\right\|_{0}\right), \\
\sup _{\left(\mathbf{v}^{h}, q^{h}\right) \in \mathbf{V}^{h} \times S^{h}} \frac{Q_{0, h}^{ \pm}\left(\mathbf{v}^{h}, q^{h} ; \mathbf{u}^{h}, p^{h}\right)}{\left\|\mathbf{v}^{h}\right\|_{1}+\left\|q^{h}\right\|_{0}}>0 &
\end{aligned}
$$

for all $\left(\mathbf{u}^{h}, p^{h}\right) \in \mathbf{V}^{h} \times S^{h}$.

Proof. We recall that the complementary plus and minus forms define equivalent problems, and so it suffices to carry the proof for just one of the forms. Here, we choose again to work with the minus form. Given a positive $\delta$, we will construct a test function $\left(\widetilde{\mathbf{v}}^{h}, \widetilde{q}^{h}\right)$ such that

$$
Q_{0, h}^{-}\left(\mathbf{u}^{h}, p^{h} ; \widetilde{\mathbf{v}}^{h}, \widetilde{q}^{h}\right) \geq C\left(\left\|\mathbf{u}^{h}\right\|_{1}+\left\|p^{h}\right\|_{0}\right)\left(\left\|\widetilde{\mathbf{v}}^{h}\right\|_{1}+\left\|\widetilde{q}^{h}\right\|_{0}\right) .
$$

To find such a function, note that definition (5.2) implies the identity

$$
\left(\nabla \mathbf{u}^{h}, \nabla \mathbf{S}^{h}\left(\nabla q^{h}\right)\right)_{0}=\left(\mathbf{u}^{h}, \nabla q^{h}\right)_{0} .
$$

Thus, if $q^{h} \in S^{h}$ is arbitrary and $\mathbf{v}_{1}^{h}=\mathbf{S}^{h}\left(\nabla q^{h}\right)$,

$$
Q_{0, h}^{-}\left(\mathbf{u}^{h}, p^{h} ; \mathbf{v}_{1}^{h}, q^{h}\right)=\left(\nabla p^{h}, \mathbf{S}^{h} \nabla q^{h}\right)_{0}+\delta h^{2}\left(-\triangle^{h} \mathbf{u}^{h}+\nabla p^{h}, \nabla q^{h}\right)_{0} .
$$

Adding and subtracting $\mathbf{Q}^{h} \nabla p^{h}$ from the last term give

$$
\begin{aligned}
Q_{0, h}^{-}\left(\mathbf{u}^{h}, p^{h} ; \mathbf{v}_{1}^{h}, q^{h}\right)= & \left(\nabla p^{h}, \mathbf{S}^{h} \nabla q^{h}\right)_{0}+\delta h^{2}\left(\left(\mathbf{I}-\mathbf{Q}^{h}\right) \nabla p^{h}, \nabla q^{h}\right)_{0} \\
& +\delta h^{2}\left(-\triangle^{h} \mathbf{u}^{h}+\mathbf{Q}^{h} \nabla p^{h}, \nabla q^{h}\right)_{0}
\end{aligned}
$$

while the orthogonality

$$
\left(\left(\mathbf{I}-\mathbf{Q}^{h}\right) \nabla p^{h}, \mathbf{Q}^{h} \nabla q^{h}\right)=0
$$


and the fact that $-\triangle^{h} \mathbf{u}^{h}+\mathbf{Q}^{h} \nabla p^{h} \in \mathbf{V}^{h}$ allow us to rewrite the last identity as

$$
\begin{aligned}
Q_{0, h}^{-}\left(\mathbf{u}^{h}, p^{h} ; \mathbf{v}_{1}^{h}, q^{h}\right)= & \left(\nabla p^{h}, \mathbf{S}^{h} \nabla q^{h}\right)_{0}+\delta h^{2}\left(\left(\mathbf{I}-\mathbf{Q}^{h}\right) \nabla p^{h},\left(\mathbf{I}-\mathbf{Q}^{h}\right) \nabla q^{h}\right)_{0} \\
& +\delta h^{2}\left(-\triangle^{h} \mathbf{u}^{h}+\mathbf{Q}^{h} \nabla p^{h}, \mathbf{Q}^{h} \nabla q^{h}\right)_{0}
\end{aligned}
$$

Next, (5.12) implies that

$$
Q_{0, h}^{-}\left(\mathbf{u}^{h}, p^{h} ; \mathbf{v}^{h}, 0\right)=\left(-\triangle^{h} \mathbf{u}^{h}+\mathbf{Q}^{h} \nabla p^{h}, \mathbf{v}^{h}\right)_{0} .
$$

Choosing $\mathbf{v}_{2}^{h}=-\delta h^{2} \mathbf{Q}^{h} \nabla q^{h}$ then gives the identity

$$
Q_{0, h}^{-}\left(\mathbf{u}^{h}, p^{h} ; \mathbf{v}_{2}^{h}, 0\right)=-\delta h^{2}\left(-\triangle^{h} \mathbf{u}^{h}+\mathbf{Q}^{h} \nabla p^{h}, \mathbf{Q}^{h} \nabla q^{h}\right)_{0} .
$$

Therefore, if $q^{h}=p^{h},(5.17)$, (5.18), and (5.15) together with the discrete negative norm characterization in (5.5) imply that

$$
\begin{aligned}
Q_{0, h}^{-}\left(\mathbf{u}^{h}, p^{h} ; \mathbf{v}_{1}^{h}+\mathbf{v}_{2}^{h}, p^{h}\right) & =\left(\nabla p^{h}, \mathbf{S}^{h} \nabla p^{h}\right)_{0}+\delta h^{2}\left(\left(\mathbf{I}-\mathbf{Q}^{h}\right) \nabla p^{h},\left(\mathbf{I}-\mathbf{Q}^{h}\right) \nabla p^{h}\right)_{0} \\
& =\left\|\nabla p^{h}\right\|_{-h}^{2}+\delta h^{2}\left\|\left(\mathbf{I}-\mathbf{Q}^{h}\right) \nabla p^{h}\right\|_{0}^{2} \geq C(\delta)\left\|\nabla p^{h}\right\|_{-1}^{2} .
\end{aligned}
$$

Since $p^{h} \in L_{0}^{2}(\Omega)$, the last inequality in combination with (2.8) gives a bound in terms of $L^{2}$ pressure norm:

$$
Q_{0, h}^{-}\left(\mathbf{u}^{h}, p^{h} ; \mathbf{v}_{1}^{h}+\mathbf{v}_{2}^{h}, p^{h}\right) \geq C_{1}(\delta)\left\|p^{h}\right\|_{0}^{2} .
$$

To complete the proof of the first weak coercivity condition, note that

$$
\begin{aligned}
Q_{0, h}^{-}\left(\mathbf{u}^{h}, p^{h} ; \mathbf{u}^{h}, 0\right) & =\left|\mathbf{u}^{h}\right|_{1}^{2}+\left(\nabla p^{h}, \mathbf{u}^{h}\right)_{0}=\left|\mathbf{u}^{h}\right|_{1}^{2}-\left(p^{h}, \nabla \cdot \mathbf{u}^{h}\right)_{0} \\
& \geq C_{P}^{2}\left\|\mathbf{u}^{h}\right\|_{1}^{2}-\sqrt{n}\left\|p^{h}\right\|_{0}\left\|\mathbf{u}^{h}\right\|_{1} \geq \frac{C_{P}^{2}}{2}\left\|\mathbf{u}^{h}\right\|_{1}^{2}-\frac{n}{2 C_{P}^{2}}\left\|p^{h}\right\|_{0}^{2} .
\end{aligned}
$$

Therefore, letting $\mathbf{v}_{3}^{h}=n^{-1} C_{1}(\delta) C_{P}^{2} \mathbf{u}^{h}$ gives

$$
Q_{0, h}^{-}\left(\mathbf{u}^{h}, p^{h} ; \mathbf{v}_{3}^{h}, 0\right) \geq \frac{C_{1}(\delta) C_{P}^{4}}{2 n}\left\|\mathbf{u}^{h}\right\|_{1}^{2}-\frac{C_{1}(\delta)}{2}\left\|p^{h}\right\|_{0}^{2},
$$

where $C_{1}(\delta)$ is the constant from (5.19). As a result,

$$
Q_{0, h}^{-}\left(\mathbf{u}^{h}, p^{h} ; \mathbf{v}_{1}^{h}+\mathbf{v}_{2}^{h}+\mathbf{v}_{3}^{h}, p^{h}\right) \geq \frac{C_{1}(\delta) C_{P}^{4}}{2 n}\left\|\mathbf{u}^{h}\right\|_{1}^{2}+\frac{C_{1}(\delta)}{2}\left\|p^{h}\right\|_{0}^{2}
$$

and the association

$$
\left(\widetilde{\mathbf{v}}^{h}, \widetilde{q}^{h}\right)=\left(\mathbf{v}_{1}^{h}+\mathbf{v}_{2}^{h}+\mathbf{v}_{3}^{h}, p^{h}\right)
$$

will fit our purpose if we can show that $\left\|\widetilde{\mathbf{v}}^{h}\right\|_{1}+\left\|\widetilde{q}^{h}\right\|_{0}$ is bounded by $\left\|\mathbf{u}^{h}\right\|_{1}+\left\|p^{h}\right\|_{0}$. Using Poincaré's inequality (2.1), we have

$$
\begin{aligned}
\left\|\widetilde{\mathbf{v}}^{h}\right\|_{1} & \leq C\left\|\nabla \widetilde{\mathbf{v}}^{h}\right\|_{0} \\
& \leq C\left(\left\|\nabla \mathbf{v}_{1}^{h}\right\|_{0}+\left\|\nabla \mathbf{v}_{2}^{h}\right\|_{0}+\left\|\nabla \mathbf{v}_{3}^{h}\right\|_{0}\right) \\
& \leq C\left(\left\|\nabla\left(\mathbf{S}^{h} \nabla p^{h}\right)\right\|_{0}+\delta h^{2}\left\|\nabla\left(\mathbf{Q}^{h} \nabla p^{h}\right)\right\|_{0}+\left\|\nabla \mathbf{u}^{h}\right\|_{0}\right) .
\end{aligned}
$$


To estimate the first term, we use the definition of $\mathbf{S}^{h}$ and Poincaré's inequality to find that

$$
\begin{aligned}
\left\|\nabla\left(\mathbf{S}^{h} \nabla p^{h}\right)\right\|_{0}^{2} & =\left(\nabla \mathbf{S}^{h} \nabla p^{h}, \nabla \mathbf{S}^{h} \nabla p^{h}\right)_{0} \\
& =\left(\nabla p^{h}, \mathbf{S}^{h} \nabla p^{h}\right)_{0}=-\left(p^{h}, \nabla \cdot \mathbf{S}^{h} \nabla p^{h}\right)_{0} \\
& \leq \sqrt{n}\left\|p^{h}\right\|_{0}\left\|\mathbf{S}^{h} \nabla p^{h}\right\|_{1} \leq C\left\|p^{h}\right\|_{0}\left\|\nabla \mathbf{S}^{h} \nabla p^{h}\right\|_{0}
\end{aligned}
$$

and, as a result,

$$
\left\|\nabla\left(\mathbf{S}^{h} \nabla p^{h}\right)\right\|_{0} \leq C\left\|p^{h}\right\|_{0}
$$

For the second term, application of the inverse inequality (2.4) twice and the fact that $\mathbf{Q}^{h}$ is bounded gives

$$
\delta h^{2}\left\|\nabla\left(\mathbf{Q}^{h} \nabla p^{h}\right)\right\|_{0} \leq \delta h C_{I}\left\|\mathbf{Q}^{h} \nabla p^{h}\right\|_{0} \leq \delta h C_{I}\left\|\nabla p^{h}\right\|_{0} \leq \delta C_{I}^{2}\left\|p^{h}\right\|_{0} .
$$

Combining all bounds shows that

$$
\left\|\widetilde{\mathbf{v}}^{h}\right\|_{1} \leq C\left(\left\|p^{h}\right\|_{0}+\left\|\mathbf{u}^{h}\right\|_{1}\right)
$$

and so we can rewrite (5.20) as

$$
Q_{0, h}^{-}\left(\mathbf{u}^{h}, p^{h} ; \widetilde{\mathbf{v}}^{h}, \widetilde{q}^{h}\right) \geq C\left(\left\|\mathbf{u}^{h}\right\|_{1}+\left\|p^{h}\right\|_{0}\right)\left(\left\|\widetilde{\mathbf{v}}^{h}\right\|_{1}+\left\|\widetilde{q}^{h}\right\|_{0}\right),
$$

which proves the first part of (5.16). To prove the second weak coercivity condition we proceed as in the proof of Theorem 4.1 and set $\mathbf{v}^{h}=-\mathbf{S}^{h} \nabla p^{h}$ and $q^{h} \equiv p^{h}$. Using definitions (5.1)-(5.3) and Lemma 5.3, we find that

$$
\begin{aligned}
Q_{0, h}^{-}\left(-\mathbf{S}^{h} \nabla p^{h}, p^{h} ; \mathbf{u}^{h}, p^{h}\right) & =\left(\mathbf{S}^{h} \nabla p^{h}, \nabla p^{h}\right)+\delta h^{2}\left(\left(\mathbf{I}-\mathbf{Q}^{h}\right) \nabla p^{h}, \nabla p^{h}\right) \\
& =\left\|\nabla p^{h}\right\|_{-h}^{2}+\delta h^{2}\left\|\left(\mathbf{I}-\mathbf{Q}^{h}\right) \nabla p^{h}\right\|_{0}^{2} \geq C(\delta)\left\|\nabla p^{h}\right\|_{0}^{2}>0 .
\end{aligned}
$$

This theorem shows that the new discrete method is stable with respect to the same norms as its continuous prototype, i.e., the natural norm on $\mathbf{H}^{1}(\Omega) \times L^{2}(\Omega)$. This valuable feature of the new method distinguishes it from the standard discrete SGLS of [18], which is stable with respect to a mesh-dependent norm.

Let us now consider the convergence of finite element solutions. The next theorem shows that the new method yields the same convergence rates as its continuous prototype with respect to the same mesh-independent norms.

ThEOREM 5.5. Let $(\mathbf{u}, p) \in \mathbf{H}_{0}^{1}(\Omega) \cap \mathbf{H}^{r+1}(\Omega) \times L_{0}^{2}(\Omega) \cap H^{s+1}(\Omega)$ denote a solution of the Stokes problem and let $\left(\mathbf{u}^{h}, p^{h}\right)$ solve (5.11). Then

$$
\left\|\mathbf{u}-\mathbf{u}^{h}\right\|_{1}+\left\|p-p^{h}\right\|_{0} \leq C\left(h^{r}\|\mathbf{u}\|_{r+1}+h^{s+1}\|p\|_{s+1}\right) .
$$

Proof. We begin by splitting the error into discrete and approximation theoretic parts:

$$
\left\|\mathbf{u}-\mathbf{u}^{h}\right\|_{1}+\left\|p-p^{h}\right\|_{0} \leq\left(\left\|\mathbf{u}_{I}^{h}-\mathbf{u}^{h}\right\|_{1}+\left\|p_{I}^{h}-p^{h}\right\|_{0}\right)+\left(\left\|\mathbf{u}-\mathbf{u}_{I}^{h}\right\|_{1}+\left\|p-p_{I}^{h}\right\|_{0}\right) .
$$

Since the interpolation error is of optimal order, to prove the theorem it suffices to 
estimate the discrete error. Using (5.16),

$$
\begin{aligned}
C(\delta) & \left(\left\|\mathbf{u}_{I}^{h}-\mathbf{u}^{h}\right\|_{1}+\left\|p_{I}^{h}-p^{h}\right\|_{0}\right) \leq \sup _{\left(\mathbf{v}^{h}, q^{h}\right) \in \mathbf{V}^{h} \times S^{h}} \frac{Q_{0, h}^{ \pm}\left(\mathbf{u}_{I}^{h}-\mathbf{u}^{h}, p_{I}^{h}-p^{h} ; \mathbf{v}^{h}, q^{h}\right)}{\left\|\mathbf{v}^{h}\right\|_{1}+\left\|q^{h}\right\|_{0}} \\
& \leq \sup _{\left(\mathbf{v}^{h}, q^{h}\right) \in \mathbf{V}^{h} \times S^{h}} \frac{Q_{0, h}^{ \pm}\left(\mathbf{u}-\mathbf{u}^{h}, p-p^{h} ; \mathbf{v}^{h}, q^{h}\right)+Q_{0, h}^{ \pm}\left(\mathbf{u}_{I}^{h}-\mathbf{u}, p_{I}^{h}-p ; \mathbf{v}^{h}, q^{h}\right)}{\left\|\mathbf{v}^{h}\right\|_{1}+\left\|q^{h}\right\|_{0}} \\
& \leq \sup _{\left(\mathbf{v}^{h}, q^{h}\right) \in \mathbf{V}^{h} \times S^{h}} \frac{Q_{0, h}^{ \pm}\left(\mathbf{u}-\mathbf{u}^{h}, p-p^{h} ; \mathbf{v}^{h}, q^{h}\right)}{\left\|\mathbf{v}^{h}\right\|_{1}+\left\|q^{h}\right\|_{0}}+C\left(\left\|\mathbf{u}-\mathbf{u}_{I}^{h}\right\|_{1}+\left\|p-p_{I}^{h}\right\|_{0}\right) \\
& \leq \sup _{q^{h} \in S^{h}} \frac{\delta h^{2}\left(-\triangle \mathbf{u},\left(\mathbf{Q}^{h}-\mathbf{I}\right) \nabla q^{h}\right)_{0}}{\left\|q^{h}\right\|_{0}}+C\left(h^{r}\|\mathbf{u}\|_{r+1}+h^{s+1}\|p\|_{s+1}\right),
\end{aligned}
$$

where to obtain the last bound we have used (5.14) in Lemma 5.2 and (2.2) and (2.3). From (5.14), it easily follows that

$$
\sup _{q^{h} \in S^{h}} \frac{\delta h^{2}\left(-\triangle \mathbf{u},\left(\mathbf{Q}^{h}-\mathbf{I}\right) \nabla q^{h}\right)_{0}}{\left\|q^{h}\right\|_{0}} \leq C h^{r}\|\mathbf{u}\|_{r+1}
$$

This means that the discrete error is of optimal order, i.e.,

$$
\left\|\mathbf{u}_{I}^{h}-\mathbf{u}^{h}\right\|_{1}+\left\|p_{I}^{h}-p^{h}\right\|_{0} \leq C\left(h^{r}\|\mathbf{u}\|_{r+1}+h^{s+1}\|p\|_{s+1}\right)
$$

and since the interpolation error is of the same order, (5.21) immediately follows.

6. Concluding remarks. Using the notion of continuous prototypes, we formulated a new absolutely stable method for the Stokes problem. The new method is a close relative of the standard pressure-Poisson stabilized method of [18] in the sense that they share the same continuous prototype.

However, the two methods differ in several important aspects. The new formulation is weakly inconsistent in the sense that, although being strictly speaking not consistent, it still leads to optimal error estimates for all $C^{0}$ finite element subspaces, including the lowest order piecewise linear case. In contrast, the standard method not only is not consistent for piecewise linear approximations (because the Laplace operator annihilates the linear velocity field in (3.17) and (3.18)) but also results in errors that do not vanish with vanishing grid sizes; i.e., there remains an error proportional to the parameter $\delta$. Furthermore, the new method is stable with respect to the norm on $\mathbf{H}^{1}(\Omega) \times L^{2}(\Omega)$, while the standard method is stable with respect to a mesh-dependent norm that is not equivalent to the norm on $\mathbf{H}^{1}(\Omega) \times L^{2}(\Omega)$.

Implementation of the new method requires evaluation of the discrete operator $-\triangle^{h}$. Given a finite element function $\mathbf{u}^{h} \in \mathbf{V}^{h}$, the coefficients $\overrightarrow{\mathbf{z}}$ of $\mathbf{z}^{h}=-\triangle^{h} \mathbf{u}^{h}$ can be determined from definition (5.1) by solving the linear system

$$
\mathbb{M} \overrightarrow{\mathbf{z}}=\overrightarrow{\mathbf{r}} .
$$

$\mathbb{M}$ is a mass matrix that can be assembled in the usual manner and $\overrightarrow{\mathbf{r}}$ is a vector with components

$$
\overrightarrow{\mathbf{r}}_{i}=\left(\nabla \mathbf{u}^{h}, \nabla \boldsymbol{\phi}_{i}^{h}\right)_{0},
$$

where $\left\{\boldsymbol{\phi}_{k}^{h}\right\}_{k=1}^{N}$ is a nodal basis for $\mathbf{V}^{h}$. In practical computations, $\mathbb{M}$ can be replaced by a lumped mass matrix or local projection. 
While computation of $-\triangle^{h}$ may seem as an additional overhead compared to the implementation of the standard method, it is well worth the effort thanks to the improved accuracy, especially when piecewise linear finite elements are used, and the guaranteed absolute, mesh-independent stability of the new method. It should be mentioned that essentially the same auxiliary problem, involving the inversion of a mass matrix, arises in standard stabilized methods with improved consistency; see [19]. These methods aim to restore the loss of consistency caused by the annihilation of all second order derivatives in the element residual when piecewise linear elements are used. The idea of [19] is to apply an $L^{2}$ projection to the first derivative of the finite element solution before the application of the second derivative so as to avoid its annihilation. Specialized to our context, this method can be viewed as providing an alternative definition for the discrete Laplace operator. Instead of the operator $-\triangle^{h}: \mathbf{H}_{0}^{1}(\Omega) \mapsto \mathbf{V}^{h}$ used in our method, they use the operator $-\triangle_{A}^{h}: \mathbf{V}^{h} \mapsto \mathbf{L}^{2}(\Omega)$ defined by

$$
-\triangle_{A}^{h}=-\nabla \cdot\left(\mathbf{Q}^{h} \nabla \mathbf{u}^{h}\right) .
$$

Let us conclude by noting that an important open question that remains to be answered is whether or not the absolute stability of the continuous SGLS prototype is inherited by other members of this class. It seems particularly worthwhile to exploit extensions of our analysis to an SGLS method defined using the alternative discrete operator (6.1) and to the original pressure-Poisson method of [18] which, as we recall, behaves numerically just like an absolutely stable formulation. Extending our results to discontinuous pressure spaces would also be valuable.

\section{REFERENCES}

[1] C. Baiocchi and F. Brezzi, Stabilization of unstable numerical methods, in Proceedings of Problemi attuali dell' analisi e della fisica matematica, Taormina, Rome, 1992, pp. 59-63.

[2] T. Barth, P. Bochev, M. Gunzburger, and J. Shadid, A taxonomy of consistently stabilized finite element methods for the Stokes problem, SIAM J. Sci. Comput., 25 (2004), pp. 15851607.

[3] M. Behr, L. Franca, and T. Tezduyar, Stabilized finite element methods for the velocitypressure-stress formulation of incompressible flows, Comput. Methods Appl. Mech. Engrg., 104 (1993), pp. 31-48.

[4] P. Bochev And M. GunzBurger, Finite element methods of least-squares type, SIAM Rev., 40 (1998), pp. 789-837.

[5] J. Bramble, R. Lazarov, and J. Pasciak, A Least Squares Approach Based on a Discrete Minus One Inner Product for First Order Systems, Technical Report 94-32, Math. Sci. Institute, Cornell University, Ithaca, NY, 1994.

[6] J. Bramble and J. Pasciak, Least-squares methods for Stokes equations based on a discrete minus one inner product, J. Comput. Appl. Math., 74 (1996), pp. 155-173.

[7] F. BREzzI, On existence, uniqueness and approximation of saddle-point problems arising from Lagrange multipliers, RAIRO Modél. Math. Anal. Numér., 21 (1974), pp. 129-151.

[8] F. Brezzi and J. Douglas, Stabilized mixed methods for the Stokes problem, Numer. Math., 53 (1988), pp. 225-235.

[9] Z. Cai And J. Douglas, Stabilized finite element methods with fast iterative solution algorithms for the Stokes problem, Comput. Methods Appl. Mech. Engrg., 166 (1998), pp. 115-129.

[10] P. Ciarlet, Finite Element Methods for Elliptic Problems, North-Holland, Amsterdam, 1978, reprinted as Classics Appl. Math. 40, SIAM, Philadelphia, 2002.

[11] J. Douglas And J. Wang, An absolutely stabilized finite element method for the Stokes problem, Math. Comp., 52 (1989), pp. 495-508.

[12] M. Fortin And R. Glowinski, Augmented Lagrangian Methods: Applications to Numerical Solution of Boundary Value Problems, Stud. Math. Appl. 15, North-Holland, Amsterdam, 1983.

[13] L. Franca, S. Frey, and T. Hughes, Stabilized finite element methods: I. Application to the advective-diffusive model, Comput. Methods Appl. Mech. Engrg., 95 (1992), pp. 253-276. 
[14] L. Franca And R. Stenberg, Error analysis of some Galerkin least-squares methods for the elasticity equations, SIAM J. Numer. Anal., 28 (1991), pp. 1680-1697.

[15] V. Girault and P. Raviart, Finite Element Methods for Navier-Stokes Equations, SpringerVerlag, Berlin, 1986.

[16] M. Gunzburger, Finite Element Methods for Viscous Incompressible Flows, Academic, Boston, 1989.

[17] T. Hughes and L. Franca, A new finite element formulation for computational fluid dynamics: VII. The Stokes problem with various well-posed boundary conditions: Symmetric formulations that converge for all velocity pressure spaces, Comput. Methods Appl. Mech. Engrg., 65 (1987), pp. 85-96.

[18] T. Hughes, L. Franca, and M. Balestra, A new finite element formulation for computational fluid dynamics: V. Circumventing the Babuska-Brezzi condition: A stable PetrovGalerkin formulation of the Stokes problem accommodating equal-order interpolations, Comput. Methods Appl. Mech. Engrg., 59 (1986), pp. 85-99.

[19] K. Jansen, S. Collis, C. Whiting, And F. Shakib, A better consistency for low-order stabilized finite element methods, Comput. Methods Appl. Mech. Engrg., 174 (1999), pp. 153170 . 